\title{
Primary healthcare professionals' perspectives on patient help-seeking for lung cancer warning signs and symptoms: a qualitative study
}

\author{
Mohamad M. Saab ( $\nabla$ msaab@ucc.ie) \\ University College Cork \\ Michelle O'Driscoll \\ University College Cork \\ Serena FitzGerald \\ University College Cork \\ Laura J. Sahm \\ University College Cork \\ Patricia Leahy-Warren \\ University College Cork \\ Brendan Noonan \\ University College Cork \\ Caroline Kilty \\ University College Cork \\ Noreen Lyons \\ Cork University Hospital \\ Heather E. Burns \\ Health Service Executive \\ Una Kennedy \\ Health Service Executive \\ Aine Lyng \\ Health Service Executive \\ Josephine Hegarty \\ University College Cork
}

\section{Research Article}

Keywords: Early detection, focus group, general practitioners, help-seeking behavior, lung cancer, nurses, pharmacists, primary care, qualitative research

Posted Date: January 18th, 2022

DOI: https://doi.org/10.21203/rs.3.rs-1260369/v1

License: (c) This work is licensed under a Creative Commons Attribution 4.0 International License. Read Full License 


\section{Abstract \\ Background}

Lung cancer is the leading cause of cancer incidence and mortality worldwide. Prompt patient help-seeking for signs and symptoms suggestive of lung cancer is crucial for early referral, diagnosis, and survivorship. However, patients diagnosed with lung cancer tend to delay help-seeking. This qualitative study explored perceived barriers to patient help-seeking and strategies to enhance help-seeking for lung cancer warning signs and symptoms from the perspective of primary healthcare professionals.

\section{Methods}

Semi-structured focus groups and individual interviews were conducted with 36 primary healthcare professionals. Data were collected via videoconferencing. Inductive thematic analysis was conducted.

\section{Results}

The following two themes were created from the data: (i) perceived barriers to patient help-seeking for signs and symptoms of concern and (ii) facilitating early patient presentation for signs and symptoms of concern. Some participants believed that the high cost of a general practitioner visit, long waiting times, and previous bad experiences with the healthcare system would deter patients from seeking help for symptoms of lung cancer. Perceived patient-related barriers to help-seeking related to the different emotions associated with a potential cancer diagnosis as well as embarrassment and guilt felt by smokers. Socio-demographic factors such as drug use, homelessness, living in rural areas, and being male and older were also perceived to impede patient help-seeking. The negative impact of the COVID-19 pandemic on cancer help-seeking also featured strongly. Participants recommended several strategies to enable patients to seek help for symptoms of concern including targeted educational campaigns focussing on symptoms (e.g., cough) rather than behaviours (e.g., smoking), accessible and free health services, and using patients' support networks.

\section{Conclusions}

Patient-related and healthcare system-related barriers to help-seeking for lung cancer warning signs and symptoms include cost of healthcare, cancer fear, and various socio-demographic factors. Increased awareness and early patient help-seeking for symptoms of concern can be achieved through targeted patient education, national campaigns, the use of community support networks, and free and accessible screening services.

\section{Background}

With an estimated 2.2 million new cases and 1.8 million deaths in 2020, lung cancer (LC) remains the leading cause of cancer incidence and mortality in men and women in 185 countries [1]. By 2040, these figures are projected to increase to 3.63 million new cases and 3.01 million deaths [2]. In the Republic of Ireland, LC is the fourth most diagnosed invasive cancer with 1,503 men and 1,250 women diagnosed each year. However, LC remains the leading cause of cancer mortality, with 1,039 men and 845 women dying from it annually [3]. LC incidence is projected to increase by $131 \%$ for males and 105\% for females by 2045 in the Republic of Ireland [4].

Early signs and symptoms of LC include a new cough, changes in an existing cough, and shortness of breath [5]. Haemoptysis is strongly associated with advanced LC but occurs in only a fifth of patients diagnosed with LC [6]. LC can also be asymptomatic until systematic symptoms occur, including unplanned weight loss and fatigue, which are often 
associated with advanced disease [7]. Therefore, the symptom signature of LC is broad [8], which may contribute to delay in patient presentation and subsequent LC diagnosis.

Prompt and early patient help-seeking for signs and symptoms suggestive of LC is crucial for prompt specialist referral, diagnosis, and survivorship. However, patients diagnosed with LC experience significant delay between symptom onset and initiation of treatment. This has detrimental effects on timely diagnosis, quality of life, cost of healthcare, and patients' eligibility for curative treatment [6,9]. In addition to lack of symptom awareness and symptom misappraisal among patients, several healthcare-related barriers to patient help-seeking and early detection of LC exist such as poor patienthealthcare professional (HCP) relationship and lack of healthcare access $[8,10,11]$. LC stigma also impacts negatively on help-seeking, leading to a median waiting time of 41 days from symptom onset to medical help-seeking [12]. COVID-19 also plays a detrimental role in cancer help-seeking and diagnosis [13].

While recent studies have explored LC help-seeking from the perspective of at-risk populations [11, 14], there has been very little research conducted amongst primary HCPs who play a key role in advising and referring patients who present to them with signs and symptoms suggestive of LC, particularly during the COVID-19 pandemic. Therefore, this study explored perceived barriers to patient help-seeking and strategies to enhance help-seeking for LC warning signs and symptoms from the perspective of primary HCPs.

\section{Methods}

\section{Design}

A qualitative descriptive design was used [15]. The 21-item Standards for Reporting Qualitative Research (SRQR) checklist was used to report this study and maintain an audit trail [16].

\section{Participants and settings}

Participation of primary HCPs (i.e., general practitioners [GPs], public health nurses [PHNs], practice nurses [PNs], and community pharmacists [CPs]) working in the Republic of Ireland was sought using non-probability purposive and snowball sampling. Various professional bodies were contacted by e-mail and asked to circulate to their members the study invitation letter, poster, and a link where primary HCPs registered their interest in participating. In addition, CPs were invited to participate during a LC symposium and via e-mail communications from colleagues.

\section{Data collection}

This study received ethical approval. Primary HCPs were provided with a study information leaflet. They were then asked to electronically sign informed consent and complete an eight-item sociodemographic questionnaire. Data were collected between February and April 2021. Due to the COVID-19 pandemic, all study documents were completed and returned electronically, and data were collected via videoconferencing. Participants were invited to participate either in focus groups or in individual interviews to enable the participation of primary HCPs who have busy schedules, particularly during the height of the COVID-19 vaccination rollout. Of note, the combination of two or more qualitative data collection methods is known to enhance data richness, depth, and trustworthiness [17].

A semi-structured interview guide was used (Table 1). Individual interviews and focus groups were facilitated by researchers (MMS, MO'D, LJS, CK) who have extensive expertise in qualitative research. Participants were also invited to comment on posters and leaflets from two National Health Service (NHS) patient-focussed LC awareness campaigns in England (Be Clear on Cancer; https://www.nhs.uk/be-clear-on-cancer/symptoms/lung-cancer) and Scotland (Get Checked Early; https://getcheckedearly.org/lung-cancer). Individual interviews and focus groups were audio-recorded. Each participant received a €20 gift voucher. 


\section{I would like you to reflect on help-seeking for lung cancer:}

\section{a. What do you think would stop a person from seeking help from a GP/public health nurse/practice nurse/community pharmacist for symptoms indicative of lung cancer?}

\section{b. What do you think would encourage a person to seek help from a GP/public health nurse/practice nurse/community pharmacist for symptoms indicative of lung cancer?}

2. Last year, we spoke to several individuals who were at risk for lung cancer. A number of these individuals refused to seek help from their GP due the (i) cost of a GP visit, (ii) previous bad experiences with the healthcare system, (iii) long waiting time to get a GP appointment, and (iv) some GPs' perceived negative attitudes towards smokers (e.g., shaming and blaming everything on smoking).

a. What do you think can be done to address such barriers?

b. Some participants indicated that as a first step they would go to their local pharmacy and buy a cough syrup. What are your views on this? (probes: opportunity to discuss the 'alarm' symptoms).

3. Share with participants the (i) "Be Clear on Cancer" poster, leaflet and symptom checker card and (ii) "Detect Cancer Early" poster and leaflet, give them 5-10min to go over those, then ask:

a. How did you find the interventions? (probes: format, colour, information, celebrity, personal stories)

b. Do you think these resources would be useful to your patients? If so, which one(s)? Why?

\section{Data analysis}

Individual interviews and focus groups were transcribed verbatim. Data were analysed using inductive thematic analysis [18]. Data analysis was iterative and began after the first interview, such that, analysis of early interviews informed the content of subsequent interviews. Excerpts from participants were extracted and shortened into codes. A coding sheet was then created with codes in one column and participant experts in a second column. Data from each participant group (i.e., GPs, PHNs, PNs, and CPs) were coded individually by one researcher (MO'D). All codes were cross-checked for accuracy, collapsed, and refined in agreement with the full researcher team. Data source triangulation was then performed by two researchers (MMS, MO'D), which involved collating all the codes and exploring similarities and differences in the data. Subthemes linking the various codes were generated and cross-checked against participants' excerpts. Similar sub-themes were then grouped into themes.

\section{Trustworthiness}

Audio-recorded memos were kept by the researchers (MMS, MO'D, LJS, CK, HEB, UK, ÁL) after each individual interview and focus group. This reflexive process helped enhance data confirmability and enabled the researchers to identify the key themes and specify areas that warranted further exploration in subsequent interviews. Dependability was established by having the research team cross-check the coding process and agree on the analysis. Transferability was enhanced by providing an audit trail and selecting a heterogenous sample of primary HCPs working across 11 Irish counties. The use of participants' own words to present data helped improve data credibility and the use of icebreakers prior to data collection helped enhance authenticity.

\section{Results}

\section{Sample characteristics}

Two focus groups were conducted with ten CPs, one individual interview and three focus groups were conducted with ten PHNs, two focus groups were conducted with eight PNs, and four individual interviews and one focus group were conducted with eight GPs, yielding a sample size of 36 participants. Focus groups lasted between 56 and 86 minutes and individual interviews lasted between 25 and 55 minutes. The majority or participants (80.5\%) were female and held either a bachelor's 
(30.6\%) or master's degree (30.6\%). Years of experience and time in current tole ranged widely from 1 to 36 years and 1 to 26 years respectively (Table 2 ).

Table 2

Sample characteristics $(n=36)$

\begin{tabular}{|c|c|c|}
\hline Sample characteristics & & $\mathrm{n}(\%)$ \\
\hline \multirow[t]{5}{*}{ Age range (years) } & $21-30$ & $5(13.9)$ \\
\hline & $31-40$ & $5(13.9)$ \\
\hline & $41-50$ & $13(36.1)$ \\
\hline & $51-60$ & $11(30.6)$ \\
\hline & $>60$ & $2(5.6)$ \\
\hline \multirow[t]{2}{*}{ Gender } & Female & $29(80.5)$ \\
\hline & Male & $7(19.5)$ \\
\hline \multirow[t]{6}{*}{ Highest level of education } & Diploma & $1(2.8)$ \\
\hline & Higher/postgraduate diploma & $6(8.3)$ \\
\hline & Bachelor's & $11(30.6)$ \\
\hline & Master's & $11(30.6)$ \\
\hline & PhD/Doctorate & $3(8.3)$ \\
\hline & Other & $4(11.1)$ \\
\hline \multirow[t]{2}{*}{ Years of experience since primary qualification } & Range: 1-36 & \\
\hline & Mean: $21.67( \pm 10.53)$ & \\
\hline \multirow[t]{5}{*}{ Current professional role } & Pharmacist & $10(27.8)$ \\
\hline & Public health nurse & $10(27.8)$ \\
\hline & Practice nurse & $8(22)$ \\
\hline & General practitioner (qualified) & $7(19.4)$ \\
\hline & General practitioner (trainee) & $1(0.3)$ \\
\hline \multirow[t]{2}{*}{ Time in current professional role (years) } & Range: 1-26 & \\
\hline & Mean: $12.3( \pm 8.8)$ & \\
\hline \multirow[t]{5}{*}{ County of work } & Cork & $18(50)$ \\
\hline & Mayo & $5(13.9)$ \\
\hline & Dublin & $4(11.1)$ \\
\hline & Waterford & $2(5.6)$ \\
\hline & Other & $7(19.6)$ \\
\hline \multirow[t]{2}{*}{ Area of work } & Urban & $22(61.1)$ \\
\hline & Rural & $14(38.9)$ \\
\hline
\end{tabular}


The following two themes were created from the data: (i) perceived barriers to patient help-seeking for signs and symptoms of concern and (ii) facilitating early patient presentation for signs and symptoms of concern (Table 3 ). 
Table 3

Study themes, sub-themes, and sample participant excerpts

\begin{tabular}{|c|c|c|}
\hline Themes & Sub-themes & Participant excerpts \\
\hline \multirow{10}{*}{$\begin{array}{l}\text { Perceived } \\
\text { barriers to } \\
\text { patient help- } \\
\text { seeking for } \\
\text { signs and } \\
\text { symptoms } \\
\text { of concern }\end{array}$} & \multirow[t]{3}{*}{$\begin{array}{l}\text { Healthcare } \\
\text { system-related } \\
\text { barriers to help- } \\
\text { seeking }\end{array}$} & $\begin{array}{l}\text { "I have people who are working. So, you're telling them you need to go to the GP... } \\
\text { and they're like, 'When am I supposed to go? I can't take time off work to go"' } \\
\text { (CP1). }\end{array}$ \\
\hline & & $\begin{array}{l}\text { "I'm suffering myself. I'm trying to find a GP. Just for myself with a GP and I've } \\
\text { asked over } 15 \text { practices around... and nothing"(PN1). }\end{array}$ \\
\hline & & $\begin{array}{l}\text { "They [family] had great faith in their GP and they were very happy with him } \\
\text { generally, but they just felt that he was not acting on the fact that she was } \\
\text { deteriorating so quick, on how they would like it to proceed. And they felt they had } \\
\text { to circumvent him"(PHN1). }\end{array}$ \\
\hline & \multirow{3}{*}{$\begin{array}{l}\text { Patient-related } \\
\text { barriers to help- } \\
\text { seeking }\end{array}$} & $\begin{array}{l}\text { "If people are smokers, they feel that perhaps I brought this on myself and I've } \\
\text { nobody to blame but myself. So, bury their head in the sand" (PN1). }\end{array}$ \\
\hline & & $\begin{array}{l}\text { "Our patients are very busy. Their heads and their lives are very full just running } \\
\text { around sorting stuff out...they literally haven't got the bandwidth for contemplating } \\
\text { something major like that [cancer diagnosis]. So, in contrast to maybe better-off } \\
\text { areas where you get the worried well coming in with every little symptom, our } \\
\text { patients, they just don't want to think about it, so they just act like it's not } \\
\text { happening... then it's up to us to pick it up..." (GP5). }\end{array}$ \\
\hline & & $\begin{array}{l}\text { "If you think of a typical pharmacy, not being stereotypical, but they might be } \\
\text { generally perceived as being more female places rather than male places. The } \\
\text { male part of the pharmacy might be stuck in a corner...you might have one shelf if } \\
\text { you're lucky and then the rest of the pharmacy is maybe cosmetics. So maybe } \\
\text { older people, older men, for example, they may be coming in to get their } \\
\text { prescription, something over the counter, maybe the odd thing in that men's } \\
\text { section and that would be it. They wouldn't necessarily have any other reason to } \\
\text { linger in a pharmacy, to browse, and maybe, there's not those opportunities to } \\
\text { pluck up the courage to have that conversation maybe with their pharmacy or } \\
\text { pharmacist..."(CP2). }\end{array}$ \\
\hline & \multirow[t]{4}{*}{$\begin{array}{l}\text { The impact of the } \\
\text { COVID-19 } \\
\text { pandemic on } \\
\text { patient help- } \\
\text { seeking }\end{array}$} & $\begin{array}{l}\text { "I've so many patients who are just getting antibiotic prescriptions emailed in and } \\
\text { it's the patients who aren't going to the GP because they're supposed to be } \\
\text { cocooning and they're not even getting seen. So, they're not even having their } \\
\text { chest listened to and they're having antibiotic prescriptions sent over and I'm not } \\
\text { even seeing them because there's a family member coming in to pick it up. So, I } \\
\text { think that's a real barrier" (CP1). }\end{array}$ \\
\hline & & $\begin{array}{l}\text { "The pandemic has been a big problem because I was dealing with somebody on } \\
\text { the phone, and I just took the notion of getting them in because I know him to be a } \\
\text { tricky customer and he had lost } 10 \text { kilos. He never mentioned that [over the } \\
\text { phone]"(GP5). }\end{array}$ \\
\hline & & "They [patients] felt that GPs weren't accessible anymore in this last year" (PN2). \\
\hline & & $\begin{array}{l}\text { "Maybe it's only applicable in COVID, but the fact that some people are still afraid } \\
\text { to go to a pharmacy, especially people presenting with coughs in a pharmacy, } \\
\text { even having someone beside them at the counter kind of hear that they have a } \\
\text { cough might kind of feel 'oh no, everyone thinks I have COVID"'(CP2). }\end{array}$ \\
\hline
\end{tabular}

$\mathrm{CP}=$ Community Pharmacist; GP=General Practitioner; $\mathrm{PHN}=$ Public Health Nurse; PN=Practice Nurse. 


Themes Sub-themes Participant excerpts

Facilitating early patient presentation for signs and symptoms of concern
Recommendations to promote patient help-seeking

"The home helps are quite good. You've a lot of people living alone in our area and the only one that they see or may be in contact with that they'd confide in. The family obviously would be in contact, but they maybe God knows where. They're not on the ground. And I find home helps are brilliant. They would ring up and they're saying that they are a little bit concerned seemingly...even people that actually deliver the meals on wheels. That sounds probably a little bit mad, but like that's the only port of contact for a lot of these people living on their own. But they seem to be on the ball, they're on the alert a lot...their role is nearly underestimated as well at times" (PN2).

"In Ireland, a lot of the advertisements you see on television about cancer, a lot of them are about smoking and stop smoking. 'Daddy, that's the last cigarette you said you'd have.' And it's not necessarily that says 'don't be afraid. Get checked out.' And maybe if it was not so pointed, maybe more people would go, 'Oh, well, do you know what? I have had a cough for three weeks. I don't smoke. Maybe I should go to the doctor.' Rather than saying, 'Oh, well, I'm smoking, so I deserve to have that cough for three weeks'" (CP1).

"What we did when we started, it is we sent out a message to all of our patients in that category and we offered them a free screening service, FREE in big letters because people like to get things for free" (PN1).

Perspectives on previous patientfocussed campaigns

\section{Be Clear on Cancer}

"You're not wasting anyone's time. That's a very useful point actually. That's really good. Your GP wants to see you. There's lots that can be done to treat lung cancer these days, especially if it's found early" (GP1).

"Be clear on cancer. That really stood up, very clearly for me and this, I did not say it for the patient, but I was raising alarm for him and he saw that ad or he saw that campaign, it gets him more aware. You need to see the doctor. I like that one. Be clear on cancer" (CP1).

"I like the poster. I find the leaflet, although I find it interesting and I think medical people would read through it, I think it's actually quite wordy for people who aren't medically minded" (PHN1).

"I liked the green...I think green is a colour of calm, but I thought it was a little bit long" (PN2).

"The card for the wallet I'd say will be in there and that's where it'll stay, to be honest with you [Laughter]"(PHN4).

\section{Detect Cancer Early}

"It is elegant. It's very nice. It's like the colour, luxurious and all of that, but the message is kind of lost" (CP1).

"If you have a cough for three weeks, see your GP. Chances are it's nothing to worry about, but a cough that doesn't clear up can be a sign of lung cancer. So it's best to get checked out and of course Alex Ferguson is targeting the men, which is fantastic"(GP1).

"The take-home message is catch it earlier and you've got a better chance. Don't get scared, get checked and it doesn't have to mean game over. It's not what it used to be. I like that one" (GP3).

"The NHS England one just has sort of random doctors on it. I've never heard of them. Can't really identify with them. This is more eye catching and it's got Fergie on it, so immediately you're going to pay attention and it's got the symptoms on it. It's nice. It's accessible. It's not overly text-driven. It's a slicker leaflet. It resonates with you better plus just simple things like the photo, it draws your attention. The other one is sort of sterile and you can't really engage with it on the same level" (GP4). 


\section{Perceived barriers to patient help-seeking for signs and symptoms of concern}

Participants identified several healthcare system-related factors and patient-related factors that they believed would deter patients from seeking help for LC warning signs and symptoms. The negative impact of the COVID-19 pandemic on helpseeking was also discussed at length.

\section{Healthcare system-related barriers to help-seeking}

In the Republic of Ireland, patients typically pay between $€ 45$ and $€ 65$ to see their GP [19]. However, adults over 70 and children under 6 are entitled to a GP visit card, which allows them to visit a GP free of charge. Also, based on individual circumstances, some patients are eligible for a medical card, which enables them to access certain health services, including GPs, for free [19]. Therefore, there was a clear divide in responses while comparing LC help-seeking among GP visit/medical card holders and self-payers. PNs and CPs found referring those who have GP visit/medical cards much easier. While they appreciated cost as an issue, PHNs noted that their services are for medical card holders; therefore, they do not see patients who need to pay for a GP visit (PHN3, PHN4). However, some GPs believed that cost would not "come into play" (GP2) if serious signs and symptoms of LC were present.

Perceived long waiting times for GP appointments or other health services was also identified as a potential barrier to helpseeking. CP2 noted that people who work during the day might not find the time to visit their GP and PN2 referred to long waiting times to get a GP appointment based on a personal experience. In contrast, GP2 stated "not buy[ing] into the waiting times argument."

Misdiagnosis or delayed LC diagnosis were also identified as healthcare system-related barriers to help-seeking. Participants seemed aware of missed diagnoses that can happen with X-rays as compared to computed tomography (CT) scans. GPs offered examples of symptoms being misappraised by other GPs. For example, "three-stone weight loss" being attributed to "morphine use" (GP4) rather than cancer. PHN1 gave examples of where delayed LC diagnosis took place because of GPs not listening to the family's concerns about a patient who "was deteriorating so quick." As a result, the family decided to "circumvent him [GP]."

\section{Patient-related barriers to help-seeking}

The possibility of a cancer diagnosis was perceived to trigger "fear of dying" (PHN1) and fear of a "quick and painful" death among patients (GP1). As a result, participants believed that patients choose to "bury their heads in the sand" (PN1) and "do not want to upset family" (GP1). A fatalistic attitude of "what happens, happens"(CP2) among some patients adds to the complexity of help-seeking. It was also highlighted that many patients "are very busy...[they] haven't got the bandwidth for contemplating something major like that [cancer diagnosis]"(GP5).

The stigma associated with smoking was discussed. It was felt that "smokers blame themselves for their symptoms" and therefore "won't bother the doctor because it's [their] own fault" (GP5). PN2 spoke of how patients "recoil" when asked whether they smoke, and are embarrassed if they do, despite being aware of the harms of smoking. CPs and PHNs acknowledged that HCPs are prone to lecturing patients about smoking and "treat[ing] them like children" (PHN3), which could lead to delayed help-seeking. They recommended that HCPs should remember that "smoking is a choice" (CP1).

Participants agreed that socioeconomic and geographic factors play a role in patient help-seeking. For instance, PN1 noted that patients in rural areas must travel for services, and often have less well-established transport links. Conversely, PN2 felt that patients in rural areas visited their GPs more often. While PHNs also acknowledged the issue of accessibility, they said that patients would not seek medical help if other factors were at play, such as fear of cancer or cost (PHN3). 
Some GPs spoke about the economic deprivation that they experience in their practices. The term "unworried unwell" was used by GP5 to describe patients from socioeconomically deprived areas who tend have multiple co-morbidities at a young age, have less educational attainment, and are less likely to engage with healthcare services. Homelessness and drug use were also perceived by GP5 to add to the complexity of help-seeking. Age and gender were also believed to play a role in help-seeking, whereby participants saw a reluctance in older men who had to be prompted by their spouses to seek help: "it is always the wife who rings up!" (PHN4), "women tend to push men to investigate" (CP1). CP2 believed that men want to "appear strong" and stated that pharmacies are "generally perceived as being more female places rather than male places." This was believed to deter older men from "pluck[ing] up the courage to have [a] conversation with their pharmacy or pharmacist" about symptoms of concern.

\section{The impact of the COVID-19 pandemic on patient help-seeking}

The COVID-19 pandemic presented unique challenges to patient help-seeking. Because cough is a high-alert symptom of COVID-19, those who were experiencing a new cough were thought by CPs and PNs to be hesitant and at times "embarrassed" (CP2) to seek help. Participants also acknowledged the fear that patients had of contracting COVID-19 in healthcare settings (e.g., GP practices, hospitals, and pharmacies) and the resulting reluctance to seek help. PHN1 gave examples of patients delaying care for so long, in other areas of health, that they needed to go "straight to A\&E [accident and emergency]" or situations ending in myocardial infarction or even death.

Lack of in-person contact with HCPs was another barrier identified by CPs due to their role in the electronic dispensing process. Medications were prescribed without the patient being physically seen by either the GP or the CP. The patients in question often being those with multiple health issues who "are supposed to be cocooning" (CP1) and who send a family member to collect the prescription on their behalf. Lack of in-person contact, and assessment was also recognised as a significant barrier by GPs and PNs. For instance, GP5 recounted a time that only by chance a physical appointment with a patient revealed significant unplanned weight loss that would have otherwise gone unmentioned, and therefore unnoticed during a telephone consultation. Conversely, GP3 recognised the extra accessibility that remote consultations have brought to clinical practice during the pandemic, and their potential long-term benefits in increasing GP access.

Participants were witness to the fact that services were paused or reconfigured, resources were redeployed, and other health issues were prioritised during the pandemic. Patients appeared to perceive COVID-19-related issues as more important than other health issues. As a result, they were slower to contact their GP about non-COVID-19-related health concerns (PN2). PN1 added that communication around this issue in the media was lacking during the height of the pandemic, thus adding to the confusion and uncertainly that patients experienced in relation to the services that continued to be available to them during the COVID-19 pandemic.

\section{Facilitating early patient presentation for signs and symptoms of concern}

Participants gave several recommendations to help promote early patient help-seeking for symptoms of concern.

\section{Recommendations to promote patient help-seeking}

Patient education was perceived to promote early help-seeking. PHN3 believed that "people don't know the symptoms or early signs of lung cancer" and CP1 thought that patient education should steer away from focusing on the underpinning behaviour (e.g., smoking) and, instead, focus on symptoms of concern (e.g., cough). Similarly, symptoms to watch for were flagged by PNs as something that patients lacked knowledge in, particularly when it comes to differentiating between symptoms of existing pulmonary diseases (e.g., chronic obstructive pulmonary diseases) and other suspicious symptoms (PN2). Patient education around early detection of LC and the resulting improvement in prognosis was also advised to reduce public misconceptions around LC being inevitable a "terminal diagnosis" (GP3). GPs and PNs also stressed the importance of educating patients about the role of the GP in LC referral and the process of further investigation. PNs

Page $10 / 17$ 
highlighted that the general population should also know about Rapid Access Lung Clinics (RALCs) which provide prompt diagnostic evaluation of patients with clinical or radiological findings suspicious for LC across eight cancer centres in the Republic of Ireland.

PHNs spoke about how breast (PHN4), cervical, and oesophageal cancers (PHN3) are "high on the agenda" in contrast to LC, due to greater media coverage. Participants felt that learnings from previous successful health awareness campaigns such as the F.A.S.T. (Facial weakness, Arm weakness, Speech problems, and Time to call 112 or 999 for an ambulance) stroke awareness campaign can be used to inform future LC awareness campaigns by using clear, concise, visual, and targeted messages.

The role of the patient's family and support networks in help-seeking was evident, with GP1 describing how some patients had been pressurised to present due to concerns from family members. PHN4 also described how patients would be more likely to "follow through" with the referral process if family support was present. Some participants expanded the concept of support and relationships to include relationships with HCPs. PNs acknowledged the role of community supports for patients, particularly those who live alone, by acknowledging the crucial role of "Home Help [government service aimed to support older people to remain in their own homes for as long as possible and to support informal carers]" and "Meals on Wheels [organisation that delivers meals to seniors, disabled, and homebound individuals for free or at low cost]" (PN2) personnel in noting deteriorations in the patient's health.

It was suggested that the approach to COVID-19 testing has come with lots of learnings, and a model upon which to base other health initiatives (GP1). Several participants recommended free and accessible lung health check/ health screening services for smokers to raise the public profile of LC (GP5), as well as universal access to CT scans. Similarly, free GP care was suggested by PHN4, while access to free-of-charge lung CT scans for all and more lung function tests were suggested by PN1.

\section{Perspectives on previous lung cancer awareness campaigns}

In general, participants preferred the Scottish campaign, describing it as "less shouty" (GP5), except for some CPs who felt that while all "the important information" (CP2) was being provided by the Scottish campaign, its delivery was not strong enough, and "was lost" (CP1). They felt that signposting to CPs as somebody to speak to with initial concerns was omitted, and this was something that should be addressed (CP1). Participants liked the emphasis placed on the message that GPs "want to see you" (GP1) in the English campaign, as well as the outlining of important symptoms (GP2) and the positive emphasis that is placed on what can be done to help (CP1). It was felt that the importance of early detection was clearly presented (CP2). Some GPs recommended including additional information on the red flags for LC in the English campaign and cough bottle use in the Scottish campaign (GP3).

In terms of visuals, some participants felt that the English campaign lacked imagery (CP2), although it was impactful as a poster campaign (CP2), as it was "big and clear" (PHN1). The leaflet could have been made more dramatic through the appropriate use of images or other visuals (GP3). Some PHNs liked the "green colour" as it felt "calm" and was "legible," while others commented that it was "not very vibrant," and the use of "brighter colours and pictures" would have been welcomed (PN1). The Scottish campaign, on the other hand, was thought to "draw your attention...eye catching and identifiable" (GP4) and was more professional in its approach, looking almost like a magazine and not like other leaflets (PHN1). CP1, however, felt that the imagery of tea and toast in the leaflet was a reminder of hospital and did not find it appropriate.

As for the use of doctors and celebrities, some GPs felt that doctors in the English campaign were "cross [angry] looking" (GP5) and the use of doctors was perceived as "sterile," while others felt that doctors seemed "benign" (CP1) and one of them was a well-known TV doctor (GP1). The use of doctors and older white patients was felt to be a limitation and gender balance was needed in the doctor representation (PHN4). Some participants, however, favoured the attempt of the English campaign to portray doctors as accessible and human and to use real and relatable patient stories (CP1). It was felt that the 
use of a celebrity (Sir Alex Ferguson) in the Scottish campaign was a worthwhile approach and would generate conversation (PHN4) and encourage patients to read the leaflet and take on board its message (PN1). Sir Ferguson was recognised by most if not all participants and was perceived as somebody who would draw people in, a friendly face, somebody "trustworthy" (GP4) and "relatable," particularly for older men (CP2). CPs, however, felt that using a celebrity ran the risk of the person not being recognised and could potentially lessen the campaign's credibility. PHN1 also noted that the celebrity may not be liked by some and using them can be "divisive."

There were mixed views about the campaign slogans and straplines. "Be Clear on Cancer" was thought to be "soft and gentle" by some (GP3), while others found it "catchy" and "clear" (PHN4). In the Scottish campaign, the use of the phrase "lung cancer doesn't have to mean game over" was welcomed, as it implied that early detection could have a positive outcome (GP3) and a LC diagnosis was not necessarily a death sentence (CP1). However, for some, the terminology "extra time" and "game over" sounded "fatalistic"(GP1, PHN3), suggesting that "you'll still die quickly" (PN1). In keeping with the Scottish campaign, the strapline "don't get scared get checked" was described as "snappy" and "very good" by some (CP2, PN2), as it recognised how people may be feeling (PHN3). However, PHN1 did not favour the use of the word "scared."

The practicality of leaflets was queried widely. The six-page leaflet of the English campaign was considered too long, particularly for patients who are "not medically minded" (PHN1) due to the risk of "information overload" (PN1) with the onepage poster providing a better amount of information than the leaflet. Some PNs and PHNs also felt that patients would get scared reading the leaflet and would not want to present as a result. This contrasted with their view of the Scottish campaign, which they felt also had too much text in the four-page leaflet, but was "less clinical" (PHN3), contained less jargon (PN2) and was more "positive and upbeat" (PN1), and therefore more appropriate to target patients as it assures that "lung cancer is not the death sentence that it used to be" (PN2). PHN4 felt that while leaflets do not get used, posters get glanced at once and then forgotten, unless they potentially were used on billboards, that "you could see while stopped in your car." Wallet/pocket card versions were perceived by PHN1 as impractical and prone to getting lost.

\section{Discussion}

This study explored perceived barriers for patient help-seeking for signs and symptoms indicative of LC and strategies to enable patients to seek help early. The cost of GP visits and long waiting times emerged as potential barriers. These findings are well documented in previous research with at-risk patients [11,14]. However, in the current study, there was no full agreement on how cost impacted on patients with LC symptoms due to the eligibility of most high-risk groups for GP visit/medical cards.

International evidence demonstrates that the probability of having a missed LC post CT scan is much lower than on chest Xray [20]. Of note, previous misdiagnosis and the resulting mistrust in the healthcare system are known as impediments to subsequent help-seeking [21]. However, the risk/benefit ratio of recommended diagnostic modalities/pathways for specific patient cohorts requires careful consideration. For example, the radiation dose associated with a CT, and the cost of delivering CT scans, far exceeds that of X-ray. Additional concerns include the potential for over diagnosis.

Participants acknowledged that perceived negative judgment by primary HCPs prevents some patients from seeking their help for symptoms of concern. This is a common phenomenon in the health literature, where one poor experience with HCPs can undermine future engagement with them thereafter. This barrier is often more pronounced for illnesses that are associated with lifestyle behaviours like smoking [11], which often triggers feelings of embarrassment, guilt, and fear of judgement among patients. Indeed, higher levels of perceived LC stigma are associated with a longer wait to seek medical help for symptoms of concern [12].

Other factors linked to help-seeking delay relate to the fear, denial, and anger that a potential cancer diagnosis can trigger among patients $[10,11]$. Such barriers were perceived to be common among men and more so among those residing in rural areas where access to healthcare services is more limited. Interestingly, participants also believed that pharmacies were

Page $12 / 17$ 
more female-friendly environments which could potentially prevent men from entering a pharmacy and seeking help from a CP. Therefore, healthcare initiatives directed at increasing men's engagement with healthcare services must consider factors that are important to men such as the individual social networks and local gender norms [22].

Participants believed that the advent of the COVID-19 pandemic has exacerbated delays in help-seeking and subsequent LC diagnosis due to fear of contacting COVID-19 in healthcare facilities, stigma surrounding a cough during the pandemic, and prioritising COVID-19-related health concerns. This was evident in a survey of 7,543 patients the UK which found that approximately half of patients who reported experiencing cancer alarm signs and symptoms did not contact their GP due to concerns about catching or transmitting COVID-19, wasting the GP's time, or putting an additional strain on healthcare services [13].

While some GPs perceived telephone consultations as helpful during the COVID-19 pandemic, other HCPs felt the lack of inperson contact could lead to missed LC diagnosis, particularly when physical changes such as unplanned weight loss cannot be readily appraised during telephone consultations. CPs also discussed the dangers of remote prescribing (e.g., antibiotics) without seeing or assessing the patient. While telephone/video consultations provide an appropriate alternative to in-person consultations in certain circumstances, several challenges exist, including limited staff training in telephone consultations, suboptimal patient-physician interaction, insufficient technical support, concerns around privacy and confidentiality, and inconsistencies in documentation [23].

Patient education was highlighted as key to early LC referral and diagnosis, particularly among PHNs who emphasised the importance of educating high-risk patients (e.g., smokers) about LC before symptoms occur. This finding is not surprising since health promotion and risk screening is at the heart of the PHN role [24]. Another strategy to raise patients' awareness of LC and engage them in early detection and referral was recommended by CPs who spoke about the importance of stressing LC symptoms rather than smoking per se. This is crucial as focusing on smoking cessation rather than LC awareness is known to trigger feelings of guilt and embarrassment among at-risk individuals who smoke, thus deterring them from seeking help for symptoms of concern [11].

In keeping with patient education, participants recommended using learnings from previous health awareness campaigns to raise the profile of LC and highlight the importance of seeking timely medical attention for LC alarm symptoms. One example cited in several focus groups was the F.A.S.T. stroke awareness campaign. This media-based campaign was broadcast in Ireland between May 2010 and June 2011 through national television and radio advertising [25]. An interrupted time series study found a significant change in A\&E attendance among patients with reported stroke symptoms and better health outcomes likely associated with this campaign. The long-term effect of this campaign, however, did not seem to be sustained [25]. Of note, stroke is a medical emergency necessitating immediate presentation to the A\&E. On the other hand, LC does not typically present as an emergency. However, there are potentially important lessons to be learned from the design and delivery of the F.A.S.T. campaign in terms of how to effectively deliver information to target audiences.

Free and accessible lung check/health screening services were also recommended to help detect LC early. Low-dose CT remains the gold standard screening test for asymptomatic high-risk cohorts in jurisdictions that offer LC screening. LC screening programmes have been shown to reduce LC mortality by up to $20 \%$ [26]. However, few countries currently operate population-based LC screening programmes, and the uptake of LC screening in countries like the USA remains low [27].

As aforementioned, participants were shown posters and leaflets from two NHS patient-focussed LC awareness campaigns. There was a clear divide between the different HCP groups. While several CPs appreciated the level of detail in the English campaign, PNs and PHNs as well as GPs commented on the length of the leaflet and the resulting risk of information overload. In contrast, the Scottish campaign was perceived as less clinical, more positive, and upbeat due to the positive slogan “Don't Get Scared Get Checked," and more appropriate to the target population. The use of a celebrity (i.e., Sir Alex Ferguson) on the cover of the Scottish campaign was also perceived as "catchy." Similar feedback was provided by at-risk individuals in a previous study who appreciated the acknowledgement of their potential fears and the positivity and clarity

Page 13/17 
of the messages in both campaigns, particularly the Scottish one [14]. Of note, the English campaign "Be Clear on Cancer" has been evaluated on several occasions and was found to significantly increase LC awareness and help-seeking and reduce the number of patients diagnosed with late-stage LC [28].

Despite ensuring trustworthiness in the conduct and reporting of this study, some limitations are worthy of note. While appropriate in the context of the current study, the use of non-probability sampling increases the risk of self-selection bias and impacts on the transferability of findings. As aforementioned, data were collected via teleconferencing; while this approach was feasible in the context of the COVID-19 pandemic, the human element of qualitative research was lacking, and there were additional challenges associated with teleconferencing such as the risk of technological difficulties. Of note, this challenge was not encountered in the present study.

\section{Conclusions}

This study offers rich insights from primary HCPs regarding barriers to patient help-seeking for signs and symptoms suggestive of LC. Some participants believed that the high cost of a GP visit for those without a medical/GP visit card, perceived long waiting times, and previous bad experiences with the healthcare system would deter patients from seeking help for symptoms of concern. Perceived patient-related barriers to help-seeking related to the different emotions that may be triggered by a potential cancer diagnosis, in addition to embarrassment and guilt felt by patients due to smoking. Certain socio-demographic factors were also perceived to impede help-seeking including drug use, homelessness, living in rural areas, and being male and older. The impact of the COVID-19 pandemic featured strongly, with many participants believing that the fear of contracting or transmitting COVID-19 reduced patient visits. While virtual GP consultations were perceived as practical during the pandemic, some participants expressed concerns around missed cancers. Participants recommended several strategies (summarised Figure 1) to enable patients to seek medical help for symptoms of concern including targeted educational campaigns/interventions focussing on symptoms (e.g., cough) rather than behaviours (e.g., smoking), accessible and free health services, and using patients' support networks to promote early help-seeking.

\section{Abbreviations}

\section{A\&E}

Accident and emergency

CP

Community pharmacist

GP

General practitioner

HCP

Healthcare professional

LC

Lung cancer

NHS

National Health Service

PN

Practice nurse

PHN

Public health nurse

SRQR

Standards for Reporting Qualitative Research

Declarations 
- Ethics approval and consent to participate: This study was performed in line with the principles of the Declaration of Helsinki. Ethical approval was granted by the Social Research Ethics Committee at University College Cork, Ireland on January 15, 2021 (Log 2020-197). All participants provided written informed consent.

- Consent for publication: Not applicable.

- Availability of data and materials: All data generated or analysed during this study are included in this published article.

- Competing interests: The authors declare that they have no competing interests.

- Funding: This work was supported by the National Cancer Control Programme, Health Service Executive, Ireland.

- Authors' contributions: MMS, HEB, UK, ÁL, and JH contributed to initiating the conception and design of the study. All authors contributed to recruitment. MMS, MO'D, LJS, and CK conducted the interviews and focus groups. MMS and MO'D analysed the data and all authors cross-checked data analysis. All authors critically revised the manuscript and approved the final manuscript.

- Acknowledgements: The authors would like to thank the public health nurses, practice nurses, general practitioners, and pharmacists who participated in this study.

\section{References}

1. Sung H, Ferlay J, Siegel RL, Laversanne M, Soerjomataram I, Jemal A, Bray F. Global cancer statistics 2020: GLOBOCAN estimates of incidence and mortality worldwide for 36 cancers in 185 countries. CA: Cancer J Clin. 2021;71(3):209-49.

2. World Health Organization International Agency for Research on Cancer, Cancer tomorrow. https://gco.iarc.fr/tomorrow/en/dataviz/isotype?cancers=15\&single_unit=100000\&types=0 [22/12/2021].

3. National Cancer Registry Ireland. Cancer in Ireland 1994-2018 with estimates for 2018-2020: Annual report of the National Cancer Registry. NCRI, Cork, Ireland, 2020.

https://www.drugsandalcohol.ie/33527/1/NCRI_Annual\%20Report_2020.pdf https://static.rasset.ie/documents/news/cancer-inequality-report-2016.pdf [22/12/2021].

4. National Cancer Registry Ireland. Cancer Incidence Projections for Ireland 2020-2045 https://www.ncri.ie/publications/cancer-trends-and-projections/cancer-incidence-projections-ireland-2020-2045 [22/12/2021].

5. Chowienczyk S, Price S, Hamilton W. Changes in the presenting symptoms of lung cancer from 2000-2017: a serial cross-sectional study of observational records in UK primary care. Br J Gen Pract. 2020;70(692):e193-9.

6. Walter FM, Rubin G, Bankhead C, Morris HC, Hall N, Mills K, Dobson C, Rintoul RC, Hamilton W, Emery J. Symptoms and other factors associated with time to diagnosis and stage of lung cancer: a prospective cohort study. $\mathrm{Br} \mathrm{J}$ Cancer. 2015;112(1):S6-13.

7. American Cancer Society, Signs and Symptoms of Lung Cancer. https://.

8. Koo MM, Hamilton W, Walter FM, Rubin GP, Lyratzopoulos G. Symptom signatures and diagnostic timeliness in cancer patients: a review of current evidence. Neoplasia. 2018;20(2):165-74.

9. World Health Organization, WHO report on cancer: setting priorities, investing wisely and providing care for all: https://www.who.int/publications-detail/who-report-on-cancer-setting-priorities-investing-wisely-and-providing-care-forall [22/12/2021].

10. Cassim S, Chepulis L, Keenan R, Kidd J, Firth M, Lawrenson R. Patient and carer perceived barriers to early presentation and diagnosis of lung cancer: a systematic review. BMC Cancer. 2019;19(1):1-4.

11. Saab MM, Noonan B, Kilty C, FitzGerald S, Collins A, Lyng Á, Kennedy U, O'Brien M, Hegarty J. Awareness and helpseeking for early signs and symptoms of lung cancer: A qualitative study with high-risk individuals. Eur J Oncol Nurs. 2021;50:101880.

12. Carter-Harris L. Lung cancer stigma as a barrier to medical help-seeking behavior: Practice implications. J Am Assoc Nurse Pract. 2015;27(5):240-5. 
13. Quinn-Scoggins HD, Cannings-John R, Moriarty Y, Whitelock V, Whitaker KL, Grozeva D, Hughes J, Townson J, Osborne K, Goddard M, McCutchan GM. Cancer symptom experience and help-seeking behaviour during the COVID-19 pandemic in the UK: a cross-sectional population survey. BMJ Open. 2021;11(9):e053095.

14. Saab MM, Kilty C, Noonan B, FitzGerald S, Collins A, Lyng Á, Kennedy U, Hegarty J. Public health messaging and strategies to promote "SWIFT" lung cancer detection: a qualitative study among high-risk individuals. J Cancer Educ. 2020;31:1-0.

15. Sandelowski M. Whatever happened to qualitative description?. Res Nurs Health. 2000;23(4):334-40.

16. O'Brien BC, Harris IB, Beckman TJ, Reed DA, Cook DA. Standards for reporting qualitative research: a synthesis of recommendations. Acad Med. 2014;89(9):1245-51.

17. Lambert SD, Loiselle CG. Combining individual interviews and focus groups to enhance data richness. J Adv Nurs. 2008;62(2):228-37.

18. Braun V, Clarke V. Using thematic analysis in psychology. Qual Res Psychol. 2006;3(2):77-101.

19. Citizens Information, GPs and private patients:

https://www.citizensinformation.ie/en/health/health_services/gp_and_hospital_services/gps_and_private_patients.html [22/12/2021].

20. Del Ciello A, Franchi P, Contegiacomo A, Cicchetti G, Bonomo L, Larici AR. Missed lung cancer: when, where, and why?. Diagn Interv Radiol. 2017;23(2):118.

21. Scott S, Walter F. Studying help-seeking for symptoms: the challenges of methods and models. Soc Personal Psychol Compass. 2010;4(8):531-47.

22. Hiebert B, Leipert B, Regan S, Burkell J. Rural men's health, health information seeking, and gender identities: A conceptual theoretical review of the literature. Am J Mens Health. 2018;12(4):863-76.

23. Hasani SA, Ghafri TA, Al Lawati H, Mohammed J, Al Mukhainai A, Al Ajmi F, Anwar H. The use of telephone consultation in primary health care during COVID-19 pandemic, Oman: perceptions from Physicians. J Prim Care.

2020;11:2150132720976480.

24. Drevdahl DJ, Canales MK. Being a real nurse: A secondary qualitative analysis of how public health nurses rework their work identities. Nurs Inq. 2020;27(4):e12360.

25. Mellon L, Hickey A, Doyle F, Dolan E, Williams D. Can a media campaign change health service use in a population with stroke symptoms? Examination of the first Irish stroke awareness campaign. Emerg Med. 2014;31(7):536-40.

26. National Lung Screening Trial Research Team. Reduced lung-cancer mortality with low-dose computed tomographic screening. N Engl J Med. 2011;365(5):395-409.

27. Jemal A, Fedewa SA. Lung cancer screening with low-dose computed tomography in the United States-2010 to 2015. JAMA Oncol. 2017;3(9):1278-81.

28. Saab MM, FitzGerald S, Noonan B, Kilty C, Collins A, Lyng Á, Kennedy U, O’Brien M, Hegarty J. Promoting lung cancer awareness, help-seeking and early detection: a systematic review of interventions. Health Promot Int. 2021.

\section{Figures}




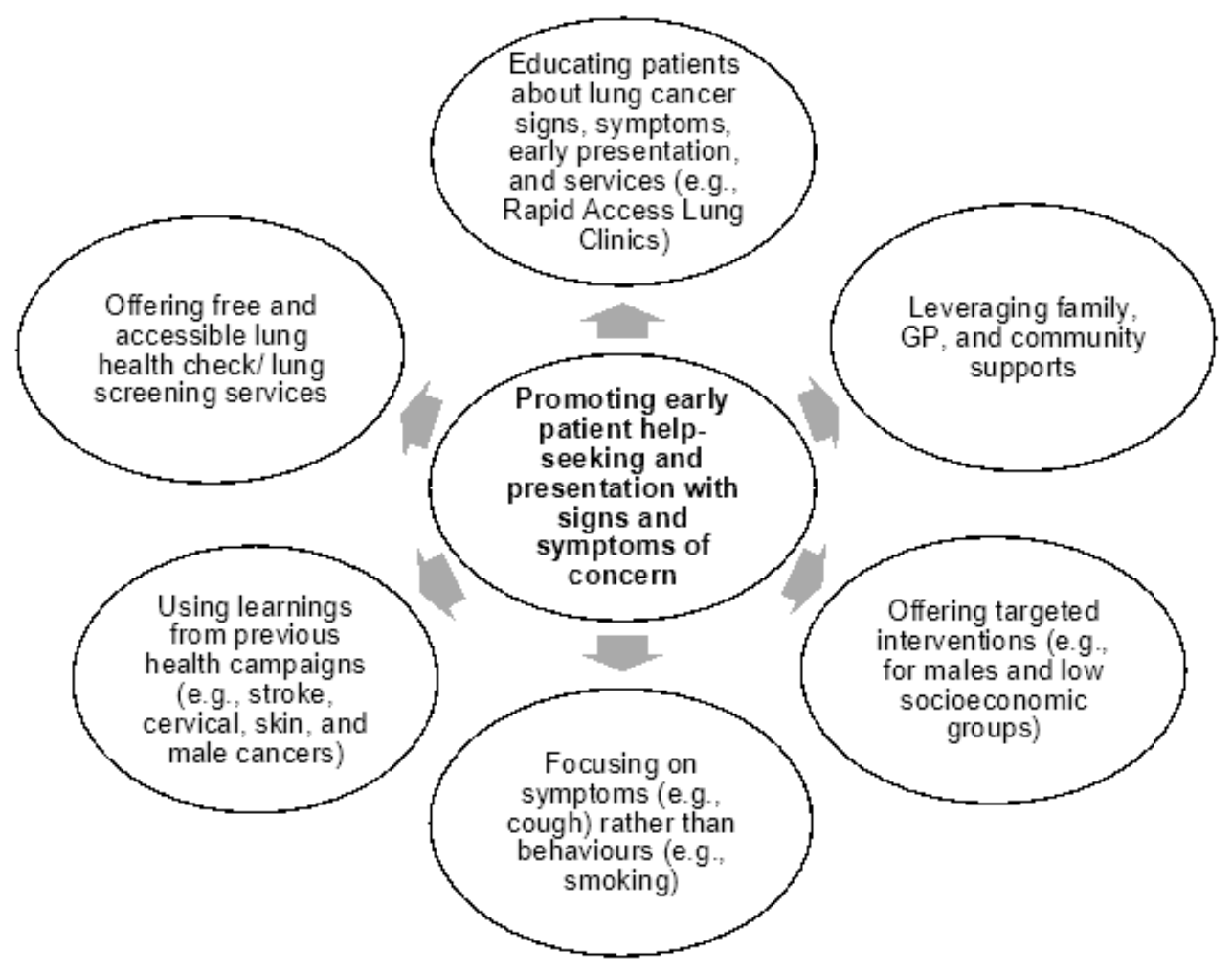

Figure 1

Summary of recommendations to promote early patient help-seeking for symptoms of concern. 